\title{
Diversidad de los helechos y licófitos de Colombia
}

\author{
José Murillo-A. \& M.T. Murillo-P.† \\ Instituto de Ciencias Naturales, Universidad Nacional de Colombia \\ Carrera 30 № 45-03, ed. 425, Bogotá, Colombia
}

\section{Correspondencia}

J. Murillo

e-mail: jcmurilloa@unal.edu.co

Recibido: 22 de mayo 2017

Aceptado: 29 agosto 2017

Publicado on-line: diciembre 2017

\begin{abstract}
Resumen
Se presenta el análisis de la diversidad de helechos y licófitos para Colombia En el país se registran 1633 especies, 44 taxones infraespecíficos y 9 híbridos, los que se distribuyen en 157 géneros y 41 familias, 184 especies pertenecen a los licófitos. Las familias con mayor número de géneros son Polypodiaceae (27), Pteridaceae (24) y Dryopteridaceae (16) y las de mayor riqueza específica son Dryopteridaceae (291 spp.), Polypodiaceae (246 spp.) y Pteridaceae (170 spp.). El género con el mayor número de especies es Elaphoglossum Schott ex J. Sm. (186 spp.). Un género (Luisma M.T. Murillo \& A.R. Sm.) y 97 especies son endémicas. La región Andina es donde se presenta la mayor diversidad, 1199 especies incluidas en 142 géneros, la región Caribe y la Orinoquía son las menos diversas. El departamento con mayor diversidad es Antioquia (869 spp.) seguido de Cundinamarca con $573 \mathrm{spp}$. Las especies con mayor área de distribución son Pityrogramma calomelanos (L.) Link, Palhihaea cernua (L.) Vasc. \& Franco y Tectaria incisa Cav. Los helechos y licófitos se encuentran desde el nivel del mar hasta cerca de los $5000 \mathrm{~m}$, no obstante, se concentran entre 1500 y $2000 \mathrm{~m}$.
\end{abstract}

Palabras clave: Distribución, diversidad, pteridófitos

\section{Abstract \\ Diversity of ferns and lycophytes of Colombia}

The diversity analysis of the ferns and lyophytes for Colombia is presented. There are 1633 species, 44 infraspecific taxa, and 9 hybrids included in 157 genera and 41 families, 184 species belong to the lycophytes. The families with the greatest number of genera are Polypodiaceae (27), Pteridaceae (24), and Dryopteridaceae (16). Families with the most number of species are Dryopteridaceae (291 spp.), Polypodiaceae (246 spp.), and Pteridaceae (170 spp.). The genus with the highest number of species is Elaphoglossum (186 spp.). One genus (Luisma) and 97 species are endemic. The greatest diversity occurs at the Andean region, where are found 1199 species belonging to 142 genera. The Caribbean region and Orinoquía are the least diverse. The department with the greatest diversity is Antioquia (869 spp.) followed by Cundinamarca with 573 spp. The species with the largest distribution range are Pityrogramma calomelanos, Palhihaea cernua and Tectaria incisa. The ferns and lycophytes are found from sea level up to $5000 \mathrm{~m}$, but they are concentrated between 1500 and $2000 \mathrm{~m}$.

Key words: Distribution, diversity, pteridophytes

\section{Introducción}

El estudio de los helechos y licófitos en
Colombia se remonta a los primeros trabajos de M.T. Murillo en 1966, cuando presentó el catálogo de las plantas del departamento de Cundinamarca. 
Posteriormente, han aparecido una variedad de trabajos principalmente de índole taxonómico, y algunos de tipo ecológico, que permiten conocer una aproximación de la diversidad florística de estos grupos de plantas en el país. Dentro de estos trabajos están las revisiones de: Blechnum L. (M.T. Murillo, 1968), Schizaea Sm. (Murillo-P., 1986), Culcitaceae, Dicksoniaceae, Lophosoriaceae, Loxsomataceae, Metaxyaceae, Plagiogyriaceae (Murillo-P., 1988a), Pteris L. (Arbeláez, 1996), Pityrogramma Link (Murillo-A. \& Murillo-P., 1999), Salpichlaena J. Sm. (Murillo-P., 2001), Luisma (Murillo-P. \& Smith, 2003), Anemia Sw. (Murillo-P. \& Murillo-A., 2004), Lellingeria A.R. Sm. \& R.C. Moran L. (Forero \& Murillo, 2010), Ceradenia L.E. Bishop (León, 2012) y Pecluma M.G. Price (Triana, 2015a). Actualmente, se están elaborando las revisiones de los géneros Cyathea Sm. (Giraldo, com pers.), Dennstaedtia Sm. (Rey et al., com pers.), Meniscium Schreb. (Forero, com. pers.), Pleopeltis Humb. \& Bonpl. ex Willd. (Camelo com. pers.) y Serpocaulon A.R. Sm (Sanin com pers.). Además, han aparecido listados de algunas familias para Colombia como los de Lycopodiaceae (Murillo-P. \& Murillo-A., 1999), Cyatheaceae (Murillo-A. \& Murillo-P., 2003) y Dryopteridaceae (León \& Murillo-P., 2005).

Dentro de los estudios regionales se cuenta con los trabajos de Murillo-P. (1997) para la sierra de La Macarena, donde se registran 180 especies. Murillo-A. et al. (2010) presenta para la región Caribe un listado de 429 especies. Murillo-P. (2000) registra 345 especies para los páramos colombianos. Murillo-A. (2004) para el Chocó biogeográfico presenta un listado de 425 especies. Rodríguez (2011) registra 768 especies para Antioquia y Rincón et al. (2014) para la Orinoquía registran 254 especies.

Entre los estudios de carácter más local se encuentran los de Giraldo \& Mejía (2002), Rodríguez (2002) y Toro (2009) en Antioquia, los de Barrera et al. (1996a), López et al. (2007), Méndez \& Murillo-A. (2014) en Boyacá, los de Sanín et al. $(2006,2008)$ en Caldas, el de Alfonso \& Murillo-A. (2000) en Caquetá, los de Murillo-P. (1988b), Murillo-P. \& Lozano (1989), Ramírez \& Macías (2007) en Cauca, los de Lellinger \& de La Sota (1972), Forero \& Gentry (1989) y Lellinger (1989) en Chocó, los de Murillo-P. (1966), Murillo-P. \& Murillo-A. (2001), Triana \& Murillo-A. (2005) y Murillo-A. et al. (2008) en Cundinamarca, y el de Ramírez \& Mendoza (2002) en Nariño.

Son pocos los estudios de índole ecológico realizados en Colombia, entre ellos, León \& Murillo-A. (2004) hace un estudio de la distribución de los helechos en diferentes paisajes de
Araracuara (Amazonas) y en esta misma región, Polanía \& Murillo-A. (2004) caracterizaron la distribución espacial de este grupo de plantas. Barrera et al. (1996b) hacen un análisis de la distribución de las epífitas y Murillo-P et al. (2002) analizan la distribución altitudinal de los helechos y licófitos al suroriente de Cundinamarca.

Un consolidado del número de especies de helechos y licófitos para Colombia fue presentado inicialmente por Murillo-P. \& Harker (1990) quienes registraron cerca de 1300 especies, y más recientemente Murillo-P. et al. (2016) presentan un listado con 1643 especies. No obstante, trabajos en Cyatheaceae (Giraldo com pers.) y Blechnum (Rojas com pers.) muestran que la composición para el país variará debido a que se están describiendo nuevas especies. En este estudio se presenta un análisis de la composición y distribución de los helechos y licófitos en Colombia.

\section{Métodos}

Este estudio se realizó con base en la información contenida en el catálogo de plantas de Colombia (Murillo-P., et al., 2015; Murillo-P. et al., 2016), las colecciones de los Herbarios COL, UPTC, TOL, PAMPLONA, publicaciones recientes de Rojas et al. (2016), Cárdenas et al. (2016) y un gran número de trabajos sobre revisiones, catálogos y especies nuevas, bibliografía que será facilitada por el primer autor. La información se registró en una base de datos, la que facilitó la obtención de cada una de las tablas presentadas. En este estudio se siguió la clasificación propuesta por PPGI (2016).

\section{Resultados}

\section{Composición}

En Colombia se registran 1633 especies, 44 taxones infraespecíficos y 9 híbridos distribuidos en 157 géneros y 41 familias, la mayoría de las cuales corresponden a los helechos, mientras que 184 especies, 10 géneros y 3 familias representan a los licófitos. Las familias con mayor número de géneros son Polypodiaceae (27), Pteridaceae (24) y Dryopteridaceae (16). Diez familias tienen entre 3 y 9 géneros y 19 solo están representadas por un género (tab. 1). Las familias con la mayor riqueza específica son Dryopteridaceae (291 spp.), Polypodiaceae (246 spp.), Pteridaceae (170 spp.) e Hymenophyllaceae (123 spp.). En pocas familias está concentrada el mayor número de especies, en 10 aparece el $79.6 \%$ (1306 spp.) de la riqueza del país, mientras que en 24 familias solo se registran 88 especies $(5.36 \%)$ (tab. 1). Los géneros con 


\begin{tabular}{|c|c|c|c|c|c|c|}
\hline Familia & No. Géneros & No. Especies & Amazonia & Andes & Pacífica & SNSM \\
\hline Polypodiaceae & 27 & 246 & 36 & 201 & 59 & 57 \\
\hline Pteridaceae & 24 & 170 & 24 & 123 & 50 & 50 \\
\hline Dryopteridaceae & 16 & 291 & 30 & 222 & 65 & 52 \\
\hline Blechnaceae & 9 & 39 & 5 & 33 & 9 & 13 \\
\hline Thelypteridaceae & 8 & 105 & 12 & 75 & 48 & 21 \\
\hline Lycopodiaceae & 8 & 83 & 8 & 67 & 10 & 16 \\
\hline Hymenophyllaceae & 6 & 123 & 38 & 82 & 51 & 22 \\
\hline Dennstaedtiaceae & 6 & 32 & 3 & 32 & 8 & 9 \\
\hline Gleicheniaceae & 4 & 30 & 3 & 24 & 12 & 9 \\
\hline Cyatheaceae & 3 & 113 & 19 & 85 & 31 & 17 \\
\hline Lindsaeaceae & 3 & 27 & 19 & 10 & 7 & 3 \\
\hline Tectariaceae & 3 & 25 & 7 & 15 & 19 & 4 \\
\hline Ophioglosaceae & 3 & 6 & 0 & 5 & 1 & 4 \\
\hline Aspleniaceae & 2 & 80 & 13 & 58 & 26 & 32 \\
\hline Marattiaceae & 2 & 22 & 7 & 10 & 11 & 5 \\
\hline Schizaceaea & 2 & 8 & 7 & 1 & 1 & 1 \\
\hline Lomariopsidaceae & 2 & 7 & 3 & 4 & 3 & 1 \\
\hline Salviniaceae & 2 & 7 & 5 & 3 & 4 & 0 \\
\hline Marsileaceae & 2 & 5 & 0 & 4 & 0 & 0 \\
\hline Woodsiaceae & 2 & 4 & 0 & 3 & 0 & 1 \\
\hline Dicksoniaceae & 2 & 3 & 0 & 3 & 1 & 3 \\
\hline Osmundaceae & 2 & 2 & 0 & 2 & 0 & 0 \\
\hline Selaginellaceae & 1 & 91 & 27 & 47 & 41 & 13 \\
\hline Athyriaceae & 1 & 54 & 6 & 45 & 22 & 11 \\
\hline Anemiaceae & 1 & 14 & 1 & 10 & 1 & 7 \\
\hline Isoetaceae & 1 & 10 & 1 & 9 & 0 & 2 \\
\hline Nephrolepidaceae & 1 & 8 & 4 & 7 & 7 & 4 \\
\hline Metaxyaceae & 1 & 4 & 3 & 1 & 1 & 0 \\
\hline Oleandraceae & 1 & 4 & 2 & 3 & 4 & 1 \\
\hline Equisetaceae & 1 & 3 & 1 & 3 & 0 & 2 \\
\hline Lygodiaceae & 1 & 3 & 1 & 1 & 2 & 2 \\
\hline Saccolomataceae & 1 & 3 & 2 & 2 & 2 & 2 \\
\hline Davalliaceae & 1 & 2 & 0 & 1 & 0 & 0 \\
\hline Psilotaceae & 1 & 2 & 0 & 1 & 1 & 0 \\
\hline Culcitaceae & 1 & 1 & 0 & 1 & 0 & 0 \\
\hline Cystopteridaceae & 1 & 1 & 0 & 1 & 0 & 1 \\
\hline Didimoclaenaceae & 1 & 1 & 1 & 1 & 1 & 1 \\
\hline Hemidyctiaceae & 1 & 1 & 0 & 1 & 1 & 1 \\
\hline Lonchitidaceae & 1 & 1 & 0 & 1 & 1 & 1 \\
\hline Loxsomataceae & 1 & 1 & 0 & 1 & 0 & 0 \\
\hline Plagiogyriaceae & 1 & 1 & 0 & 1 & 0 & 0 \\
\hline Total & 157 & 1633 & 288 & 1199 & 500 & 368 \\
\hline
\end{tabular}

Tabla 1. Número de géneros y especies por familia y distribución de las especies por las Regiones Naturales más diversas. (SNSM: Sierra Nevada de Santa Marta). Table 1. Number of genera and species by family and distribution of species by the most diverse Natural Regions. 


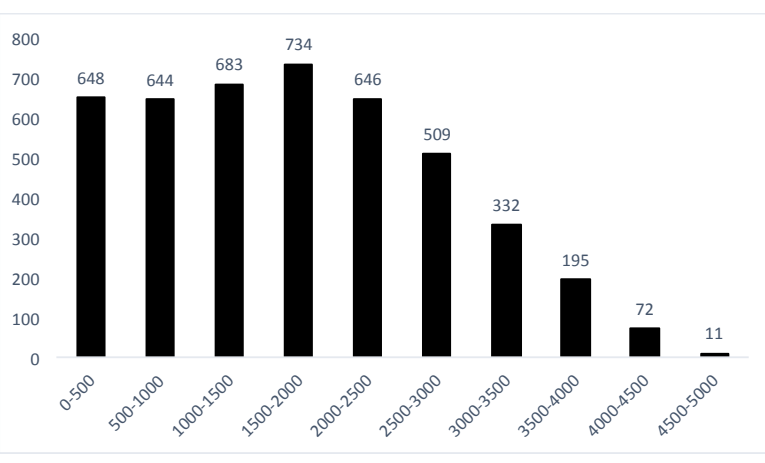

Figura 1. Distribución altitudinal de los helechos y licófitos en Colombia. Los números sobre las barras indican la cantidad de especies por rango altitudinal. Figure 1. Altitudinal distribution of ferns and lycophytes in Colombia. The numbllers on the bars indicate the number of species by altitudinal range.

mayor número de especies son Elaphoglossum (186 spp.), Cyathea (104 spp.) y Selaginella P. Beauv. (91 spp.). En solo 12 géneros se concentra cerca del 50 \% (827 spp.) de la riqueza específica y en 118 géneros se presentan entre 1 y 9 especies (21.45\%) (tab. 2).

\section{Endemismos}

En Colombia se encuentra un género endémico (Luisma), restringido al departamento de Risaralda y representado por dos colecciones depositadas en COL, y 97 especies endémicas, las que pertenecen a 39 géneros y que representan el $6 \%$ de las especies colombianas. En Elaphoglossum (12 spp.), Cyathea (10 spp.) y Phlegmariurus Holub (8 spp.) se encuentra el mayor número de especies endémicas, los otros géneros poseen menos de 6 especies, en 16 géneros hay solo una especie endémica (tab. 3). En la región Andina se encuentra la mayor cantidad de endemismos (70 spp.) seguida de la región Pacífica (21 spp.), en las restantes regiones hay menos 6 especies y en la región Caribe no se registran endemismos (tab. 4). Los departamentos con mayor número de endemismos son Antioquia (27), Boyacá (20), Chocó (20), Valle del Cauca (18) y Cundinamarca (17), en 16 departamentos hay entre 1 y 14 especies y en los restantes 11 no se presentan. 52 especies están presentes en un solo departamento y 38 solo se conocen del espécimen tipo.

\section{Distribución}

La región Andina es donde se presenta la mayor diversidad de helechos y licófitos, se registran 1199 especies incluidas en 142 géneros y 41 fami- lias, en las otras regiones se presentan menos de 502 especies, y en la región Caribe y la Orinoquía es donde se encuentra la menor diversidad 165 y 134 spp., respectivamente (tabs. 1, 5). Las familias más diversas tienen una distribución principalmente Andina (tab. 1). El departamento con mayor diversidad es Antioquia (873 spp.) seguido de Cundinamarca (574 spp.), Chocó (541 spp.), Cauca (502 spp.) y Nariño (498) (tab. 6). Los departamentos más diversos se concentran en la región Andina, Pacífica y los de menor diversidad en la Orinoquía y en algunos del Caribe. En general, las familias y los géneros son más diversos en la región Andina (tabs. 1, 2, 5). Las especies con mayor área de distribución son $P$. calomelanos, $P$ cernua y $T$. incisa. Hay 415 especies con distribución restringida, solo aparecen en un departamento.

Los helechos y licófitos se encuentran a lo largo del rango altitudinal hasta cerca de los 5000 $\mathrm{m}$, no obstante, se concentran en alturas menores de $2500 \mathrm{~m}$, con la mayor diversidad entre 1500 y 2000 m (734 spp.), en alturas superiores la diversidad disminuye (fig. 1).

\section{Especies introducidas}

En Colombia se registran seis especies naturalizadas, Christella dentata (Forssk.) Brownsey \& Jermy, Macrothelypteris torresiana (Gaudich.) Ching y Nephrolepis brownii (Desv.) Hovenkamp \& Miyam., las cuales son más ampliamente distribuidas que Pteris cretica L. Pteris vittata L., Selaginella plana (Desv. ex Poir.) Hieron. y Selaginella willdenowii (Desv. ex Poir.) Baker que se distribuyen en menos de tres departamentos. Dentro de las especies cultivadas están: Davallia canariensis (L.) Sm., Davallia fejeensis Hook., Microsorum punctatum (L.) Copel., Neoblechnum brasiliense (Desv.) Gasper \& V.A.O. Dittrich, Nephrolepis cordifolia (L.) C. Presl, Platycerium angolense Welw. ex Hook., Platycerium bifurcatum (Cav.) C. Chr., Rumohra adiantiformis (G. Forst.) Ching y Salvinia molesta D.S. Mitch.

\section{Discusión y Conclusiones}

La riqueza de helechos y licófitos se ha atribuido a diferentes factores, entre ellos la altitud y la humedad (Ferrer \& Vetaas, 2005), y en general, se ha encontrado que las regiones tropicales montañosas y con alta humedad poseen la mayor riqueza de estos grupos de plantas, con una disminución hacia las regiones árticas y desiertos (Kreft et al., 2010), no obstante, se ha propuesto que la humedad es el factor que más determina la riqueza de estas plantas (Chen et al., 


\begin{tabular}{|c|c|c|c|c|c|}
\hline Género & No. de especies & Amazonia & Andes & Pacífica & SNSM \\
\hline Elaphoglossum & 186 & 10 & 142 & 30 & 33 \\
\hline Cyathea & 104 & 18 & 73 & 27 & 16 \\
\hline Selaginella & 91 & 27 & 47 & 41 & 13 \\
\hline Asplenium & 72 & 12 & 55 & 25 & 29 \\
\hline Phlegmariurus & 65 & 1 & 52 & 7 & 11 \\
\hline Hymenophyllum & 55 & 4 & 49 & 11 & 7 \\
\hline Diplazium & 54 & 6 & 45 & 22 & 11 \\
\hline Amauropleta & 50 & 1 & 45 & 7 & 10 \\
\hline Trichomanes & 44 & 28 & 20 & 25 & 5 \\
\hline Adiantum & 41 & 9 & 20 & 20 & 12 \\
\hline Jamesonia & 34 & 0 & 32 & 0 & 7 \\
\hline Pteris & 31 & 4 & 28 & 9 & 12 \\
\hline 27 géneros & $10-26$ & & & & \\
\hline 118 géneros & 9 ó menos & & & & \\
\hline
\end{tabular}

Tabla 2. Número de especies por género y su distribución por las Regiones Naturales con mayor riqueza de helechos y licófitos. (SNSM: Sierra Nevada de Santa Marta). Table 2. Number of species by genus and their distribution by the Natural Regions with greater richness of ferns and lycophytes.

2011; Bickford \& Laffan, 2006; Kreft et al., 2010). No solo la altitud sino la humedad son factores que determinan una amplia gama de paisajes que posee Colombia, en donde se presentan cinco regiones naturales, la Andina con tres cordilleras, y en el Choco Biogeográfico y en la amazonia con una abundante precipitación y alta humedad relativa hacen de Colombia un país muy diverso paisajísticamente y con los factores más importantes para tener una gran diversidad de helechos y licófitos.

En el trabajo de Murillo-P. \& Harker (1990) apareció el primer listado de las especies colombianas de helechos y licófitos, donde se registraron cerca de 1300 especies, más recientemente Murillo-P et al. (2016) elevan el número a 1643, y con algunas correcciones en este estudio se consideran 1633 especies, las que representan cerca del $46 \%$ de la diversidad presente en América (Tryon \& Tryon, 1982) y el $6.9 \%$ de la flora vascular colombiana, la cual se estima en 23761 especies (Bernal et al., 2016). Regiones como Mesoamerica (1358 spp.; Moran \& Riba, 1995) o países altamente diversos como Ecuador (1298 spp.; Jørgensen \& León, 1999) poseen una riqueza mucho menor (tab. 6), es decir, en Colombia se encuentra entre un $16.8 \%$ y un $53.5 \%$ de mayor riqueza que en otros paises de la región, por tanto, Colombia se posiciona como el país con mayor diversidad de estos grupos vegetales en América. Cabe resaltar que a pesar de tener en Colombia un mayor número de especies, las familias más ricas son las mismas altamente diversas que en otros paises latinoamericanos (Mehltreter \& Palacios, 2002)

Los helechos y licófitos y las plantas con semillas están espacialmente muy correlacionados (Bhattarai et al., 2004; Kreft et al., 2010; Chen et al., 2011), lo que podría implicar vínculos funcionales entre todos estos grupos de plantas (Chen et al., 2011). La diversificación de los helechos polipodiodes (epífitos) fue posterior a la diversificación de las plantas con flores, lo que sucedió en el cretáceo (Schneider et al., 2004), y teniendo en cuenta la gran diversidad arbórea del país, que registra cerca de 7800 especies (Bernal et al., 2016), y la gran extensión de los bosques del país junto con la gran humedad, hacen que el hábitat que podrían ocupar los helechos y licófitos en Colombia, especialmente los epífitos, sea considerable. Todas estas condiciones podrían ayudar a explicar su gran riqueza en el país.

Los patrones de distribución muestran que tanto las familias como los géneros más diversos tienen una distribución principalmente andina, con excepción de Lindsaea Dryand ex. Sm., Trichomanes L., Salviniaceae y Schizaceae que son principalmente amazónicos, y Tectariaceae y Marattiaceae del pacífico, lo cual podría sugerir que los Andes tuvieron una gran importancia en el proceso de diversificación de los helechos y licófitos en el noroeste de Suramérica, como 


\begin{tabular}{lcc}
\hline Género & $\begin{array}{c}\text { Endémicas/ } \\
\text { colombianas }\end{array}$ & $\begin{array}{c}\% \text { de } \\
\text { endémicas }\end{array}$ \\
\hline Elaphoglossum & $12 / 186$ & 6.45 \\
Cyathea & $10 / 104$ & 9.61 \\
Phlegmariurus & $8 / 65$ & 12.3 \\
Diplazium & $6 / 54$ & 11.1 \\
Ceradenia & $5 / 24$ & 20.8 \\
Lellingeria & $4 / 18$ & 22.2 \\
Jamesonia & $3 / 34$ & 8.8 \\
Amauropelta & $3 / 50$ & 6 \\
15 géneros & 2 & \\
16 géneros & 1 & \\
\hline \multicolumn{1}{c}{39 géneros } & $97 / 1633$ & 6 \\
\hline
\end{tabular}

Tabla 3. Número de especies endémicas por género. Table 3. Number of endemic species by genus.

\begin{tabular}{lc} 
Región Natural & No. de especies \\
\hline Andina & 70 \\
Pacífica & 21 \\
Valles interandinos & 6 \\
Región Guayana y La Macarena & 4 \\
SN Santa Marta & 2 \\
Orinoquia & 3 \\
Amazonia & 1 \\
Caribe & 0 \\
\hline
\end{tabular}

Tabla 4. Número de especies endémicas por Región Natural. Table 4. Number of endemic species by Natural Region.

\begin{tabular}{lcccc}
\hline Regiones Naturales & Familias & Géneros & Especies \\
\cline { 1 - 1 } Andina & 41 & & 142 & 1199 \\
Pacífica & 31 & & 97 & 500 \\
SN Santa Marta & 32 & & 98 & 368 \\
Amazonia & 28 & & 78 & 288 \\
Valles interandinos & 26 & & 70 & 240 \\
Región Guayana y & 25 & & 76 & 215 \\
La Macarena & & & \\
Caribe & 31 & 76 & 165 \\
Orinoquia & 21 & 54 & 134 \\
\hline
\end{tabular}

Tabla 5. Distribución de los helechos y lícofitos por Regiones Naturales. Table 5. Distribution of ferns and lycophytes by Natural Regions.

\begin{tabular}{|c|c|c|c|}
\hline Departamento & $\begin{array}{c}\text { No. de } \\
\text { especies }\end{array}$ & Departamento & $\begin{array}{c}\text { No. de } \\
\text { especies }\end{array}$ \\
\hline Antioquia & 873 & Caldas & 213 \\
\hline Cundinamarca & 574 & Amazonas & 212 \\
\hline Chocó & 541 & Cesar & 146 \\
\hline Cauca & 502 & Quindio & 104 \\
\hline Nariño & 498 & Guajira & 84 \\
\hline $\begin{array}{l}\text { Valle del } \\
\text { Cauca }\end{array}$ & 469 & Guainia & 50 \\
\hline Boyacá & 468 & Bolivar & 45 \\
\hline Santander & 420 & Vichada & 44 \\
\hline Magdalena & 388 & Casanare & 35 \\
\hline Meta & 346 & Arauca & 33 \\
\hline Putumayo & 286 & Guaviare & 31 \\
\hline Huila & 282 & Córdoba & 24 \\
\hline $\begin{array}{l}\text { Norte de } \\
\text { Santander }\end{array}$ & 276 & Atlantico & 10 \\
\hline Tolima & 243 & $\begin{array}{l}\text { San Andrés y } \\
\text { Providencia }\end{array}$ & 6 \\
\hline Risaralda & 224 & Sucre & 6 \\
\hline Caquetá & 223 & & \\
\hline
\end{tabular}

Tabla 6. Número de especies por departamento. Table 6. Number of species by department.

\begin{tabular}{ll}
\hline País o región & Número de especies \\
\cline { 1 - 1 } Colombia & 1633 (Este estudio) \\
Mesoamérica & 1358 (Moran \& Riba, 1995) \\
Ecuador & 1298 (Jørgensen \& León, 1999) \\
Perú & 1200 (Smith et al., 2005) \\
Brasil & 1176 (Forzza et al., 2010) \\
Venezuela & 1155 (Hokche et al., 2008) \\
Bolivia & 1145 (Jørgensen et al., 2015) \\
México & 1008 (Mickel \& Smith, 2004) \\
Cono Sur, & 759 (Zuloaga et al. 2008) \\
América & \\
\hline
\end{tabular}

Tabla 7. Diversidad de helechos y licóficos en América. Table 7. Diversity of ferns and lycophytes in America. 
ha sido propuesto para diversos grupos de angiospermas (Hughes \& Eastwood, 2006; Chacón et al., 2012). Géneros como Ceradenia, Isoëtes L., Jamesonia Hook. \& Grev., Phlegmariurus, Melpomene A.R. Sm. \& R.C. Moran, Plagyogiria (Kunze) Mett., Loxsomopsis H. Christ son principalmente altoandinos, mientras que Adiantum L., Didymoglossum Desv., Goniopteris C. Presl, Linsdsaea, Microgramma C. Presl, Selaginella, Tectaria Cav., Trichomanes son principamnete de zonas bajas, menores de $500 \mathrm{~m}$ de altitud.

En Colombia los helechos y licófitos están ampliamente distribuidos desde el nivel del mar hasta cerca de los $5000 \mathrm{~m}$, su mayor diversidad se encuentra entre los 1500 y 2000 m, resultados similares se han encontrado en otras zonas de América (Kessler, 2000) y de África (Battarai et al., 2004). En Colombia, en alturas menores la diversidad disminuye poco (648-683 spp., fig. 1) incluso a los $2500 \mathrm{~m}$ se registran 646 especies, pero a mayor altitud la diversidad se reduce fuertemente (fig. 1), lo que resulta en una curva sesgada hacia la izquierda, contrario a lo que ocurre en estudios locales, donde la distribución es unimodal con un máximo entre 1500 y 2000 m (Kessler, 2000; Battarai et al. 2004). Al parecer, la gran diversidad paisajística y la alta humedad en las zonas bajas colombianas permiten una gran riqueza, al menos de helechos, que podrían permitir una distribución diferente que la obtenida en áreas más locales.

Estudios exhaustivos para conocer la composición y distribución de las especies de helechos y licófitos del país no se han realizado, y por tanto es probable que la riqueza en las diferentes regiones del país podría estar subestimada. En revisiones recientes en Caldas (Triana, 2015b) se propone que en el departamento hay 343 especies, no obstante, nuestros datos solo reconocen 213 spp., para el Meta (Gutiérrez et al., en prep.) se registran 53 especies más a las 348 que tenemos registradas y para Sucre (Calderón, com. pers.) eleva el número de especies de 6 a cerca de 40. Para Antioquia Rodríguez (2011) registró 768 especies, pero aquí tenemos 101 registros más, y para Boyacá Bravo (2015) registra 431 ssp. y en este estudio tenemos 38 registros más. Por lo tanto, un estudio regional más detallado permitirá tener una idea más clara de cuál es la distribución de las especies colombianas.

A pesar de tener revisiones o sinopsis de algunos de los géneros colombianos y que permiten establecer su diversidad específica en el país, géneros como Elaphoglossum (186 spp.), el más diverso de Colombia, Selaginella (91 spp.), Asplenium L. (72 spp.), Diplazium Sw. (54 spp.), Trichomanes (44 spp), Adiantum (41 spp.),
Jamesonia (33 spp.) no cuentan con una revisión, otros como Cyathea (104 spp., Giraldo com pers.), Serpocaulon (24 spp., Sanín com. pers.) y Dennstaedtia (18 spp., Chica et al. en prep.) se están revisando actualmente, no obstante, falta gran cantidad de géneros de los cuales solo se tiene un registro aproximado, sin embargo, este trabajo se apoyada con estudios de índole regional (Alfonso \& Murillo-A., 2000; Triana \& Murillo-A., 2005; Méndez \& Murillo-A., 2014; Gutierrez et al., en prep.) que ayudan no solo a definir y caracterizar los taxones sino que permiten conocer cuál es la distribución de las especies.

En cuanto al uso que se les da a los helechos y licófitos es principalmente ornamental o medicinal (Murillo-P., 1983), y dentro de las ornamentales se usan generalmente especies exóticas de Nephrolepis Schott y Platycerium Desv., pero no se tienen en cuenta el enorme potencial ornamental, que podrían tener especies de los géneros Asplenium, Adiantum, Blechnum, Cyathea y Selaginella, entre otros, afortunadamente se están cultivando en separadores de avenidas y centros comerciales, especialmente, especies arbóreas de la familia Cyatheaceae.

\section{Agradecimientos}

A los herbarios COL, UPTC, TOL, PAMPLONA y sus curadores generales por permitir la revisión de los ejemplares. A Alexander Rojas por sus comentarios al manuscrito.

\section{Referencias}

Alfonso, R. \& Murillo-A., J. (2000). "Pteridófitos de Colombia III. Los pteridófitos de la región de Araracuara (Amazonía Colombiana)". Biota Colombiana, 1, 217223.

Arbeláez, A.L. (1996). La tribu Pteridae (Pteridaceae). Bogotá: Flora de Colombia. Vol. 18. Instituto de Ciencias Naturales, Universidad Nacional de Colombia.

Barrera, E., Acosta, N., \& Murillo-P., M.T. (1996a). Helechos y afines del Santuario de Flora y Fauna de Iguaque, Boyacá, Colombia. Acta Biológica Colombiana, 3, 79-92.

Barrera, E., Barrera, A. \& Murillo-P., M.T. (1996b). Pteridófitas epífitas de la franja subandina del departamento de Cundinamarca, Colombia. Revista de la Academia Colombiana de Ciencias Exactas, Físicas y Naturales, 20, 47-55.

Bhattarai, K., Vetaas, O.R. \& Grytnes, J.A. (2004). Fern species richness along a central Himalayan elevational gradient, Nepal. Journal of Biogeography, 31, 389-400.

Bickford, S.A. \& Laffan, S.W. (2006). Multi-extent analysis of the relationship between pteridophyte 
species richness and climate. Global Ecology and Biogeography, 15, 588-601.

Bravo, W. \& Diaz, N. (2015). Estado del conocimiento de los helechos y licófitas en el departamento de Boyacá. En J. Ramírez (Ed.), Libro de resumenes VIII Congreso Colombiano de Botánica (p. 163). Manizales: Asociación Colombiana de Botánica.

Cárdenas, G. Tuomisto, H, \& Lehtonen, S. (2016). Newly discovered diversity in the tropical fern genus Metaxya based on morphology and molecular phylogenetic analyses. Kew Bulletin, 71, 1-27.

Chacón, J., Camargo de Assis, M., Meerow, A.W. \& Renner, S.S. (2012). From East Gondwana to Central America: historical biogeography of the Alstroemeriaceae. Journal of Biogeography, 39, 1806-1818.

Chen, S., Jiang, G., Ouyang, Z., Xu, W. \& Xiao, Y. 2011. Relative importance of water, energy, and heterogeneity in determining regional pteridophyte and seed plant richness in China. Journal of Systematics and Evolutions, 49, 95-107.

Ferrer, D. \& Vetaas, O.R. (2005). Pteridophyte richness, climate and topography in the Iberian Peninsula: comparing spatial and nonspatial models of richness patterns. Global Ecology and Biogeography, 14, 155165.

Forero, E. \& Gentry, A. (1989). Lista anotada de las plantas del departamento del Chocó, Colombia. Bogotá: Biblioteca José Jerónimo Triana, Instituto de Ciencias Naturales, Universidad Nacional de Colombia.

Forero, D. \& Murillo-A., J. (2010). Revisión taxonómica del género Lellingeria (Polypodiaceae) para Colombia. Caldasia, 32, 311-337.

Forzza, R., Baumgratz, J., Bicudo, C., Carvalho Jr., A., Costa, et al. (Eds.) (2010). Catálogo de plantas e fungos do Brasil. Rio de Janeiro: Andrea Jakobsson Estúdio: Instituto de Pesquisas Jardim Botânico do Rio de Janeiro

Giraldo, L.F. \& Mejía, P. (2002). Catálogo ilustrado de los helechos arbóreos de Antioquia. Medellín: Corporación Autónoma regional del dentro de Antioquia. Fundación Jardín Botánico Joaquín Antonio Uribe.

Hokche, O., Berry, P. \& Huber, O. (Eds.) (2008). Nuevo catálogo de la flora vascular de Venezuela. Caracas: Fundación Instituto Botánico de Venezuela Dr. Tobías Lasser.

Hughes, C. \& Eastwood, R. (2006). Island radiation on a continental scale: Exceptional rates of plant diversification after uplift of the Andes. PNAS, 103, 10334-10339.

Jörgensen, M., Michael, P., Nee, H. \& Beck, S. (Eds.) (2015). Catálogo de las Plantas Vasculares de Bolivia. Monographs in Systematic Botany from the Missouri Botanical Garden, 127, 1-1741.

Jørgensen, P.M. \& León-Yánez, B. (Eds.). (1999). Catalogue of the vascular plants of Ecuador. Monographs in Systematic Botany from the Missouri Botanical Garden, 75, 1-1182.

Kessler, M. (2000). Elevational gradients in species richness and endemism of selected plant groups in the central Bolivian Andes. Plant Ecology, 149, 181-193.
Kreft, H., Jetz, W., Mutke, J. \& Barthlott, W. (2010). Contrasting environmental and regional effects on global pteridophyte and seed plant diversity. Ecography, 33, 408-419.

Lellinger, D.B. \& De La Sota, E. (1972). Collecting ferns in the Chocó, Colombia. American Fern Journal, 62, 1-8.

Lellinger, D.B. (1989). The ferns and fern - allies of Costa Rica, Panamá and the Chocó. Part. 1: Psilotaceae throught Dicksoniaceae. Pteridology, 2A, 1-364.

León, A. \& Murillo-A., J. (2004). Diversidad y estructura de los pteridófios de la cuenca media del río Caquetá (Amazonía colombiana). Acta Biológica Colombiana, 9, 112-113.

León, A. \& Murillo-P., M.T. (2005). Listado de especies de la subfamilia Dryopteridoideae, tribus Dryopteridae. Rumohreae, Tectarieae (Familia: Dryopteridaceae) para Colombia. Biota Colombiana, 6, 87-100.

León, A. (2012). Ceradenia (Polypodiaceae). Bogotá: Flora de Colombia $N^{\circ}$ 19. Instituto de Ciencias Naturales. Universidad Nacional de Colombia.

López, P.M., Torres, E.P., Murillo-A., J. \& Morales, M.E. (2007). Los helechos y plantas afines de la reserva natural Ranchería, Paipa, Boyacá-Colombia. Tunja: Colección de investigación UPTC No. 11. Universidad Pedagógica y Tecnológica de Colombia.

Mehltreter, K, Palacios, M. (2002). The flora Pteridologica latinoamericana database and its use for assessing Pteridophyte diversity and biogeography. Fern Gazette, 16, 398-403.

Méndez, M. \& Murillo-A., J. (2014). Helechos y lycófitos de Santa Maria (Boyacá, Colombia). Bogotá: Serie de Guías de Campo del Instituto de Ciencias Naturales No. 14. Universidad Nacional de Colombia.

Mickel, J. T. \& Smith, A. R. (2004). The pteridophytes of Mexico. Memoirs of the New York Botanical Garden 88: 1-1054.

Moran, R.C., \& Riba, R. (Eds.). (1995). Psilotaceae a Salviniaceae. En G. Davidse, G., M. Souza, S. Knapp. (Eds.), Flora Mesoamericana (pp. 1-470). México: Volumen 1. Universidad Nacional Autónoma de México.

Murillo-A., J. (2004). Diversidad y riqueza de pteridófitos en el Chocó biogeográfico. En J. Rangel (Ed.), Colombia Diversidad Biótica IV. El Chocó biogeográfico/Costa pacífica (pp. 513-558). Bogotá: Instituto de Ciencias Naturales, Universidad Nacional de Colombia, Conservación Internacional Colombia.

Murillo-A., J. \& Murillo-P., M.T. (1999). Pteridófitos de Colombia II. El género Pityrogramma (Pteridacea). Revista de la Academia Colombiana de Ciencias Exactas, Físicas y Naturales, 27, 45-51.

Murillo-A., J. \& Murillo-P., M.T. (2003). Pteridófitos de Colombia IV: Novedades en Cyathea (Cyatheaceae). Revista de la Academia Colombiana de Ciencias Exactas, Físicas y Naturales, 27, 45-51.

Murillo-A., J., León, A. \& Polanía, C. (2002). Efecto del gradiente altitudinal en la distribución de los pteridófitos del Guavio Cundinamarca (Colombia). En O. Rangel, J. Aguirre \& G. Andrade (Eds.), Libro de resúmenes octavo congreso latinoamericano y segundo colombiano de botánica (pp. 340). Bogotá: Editorial Unibiblos. 
Murillo-A., J., Polanía, C. \& León, A. (2008). Los helechos y licófitos de la región del Guavio. Biota Colombiana, 9, 63-76.

Murillo, A., J., Orozco, N. \& Rivera, O. (2010). Los helechos y licófitos del caribe colombiano. En O. Rangel (Ed.), Colombia Diversidad Biótica XII. La Región Caribe de Colombia (pp. 333-348). Bogotá: Editorial Códice Ltda.

MURILLO-P., M.T. (1966). Pteridophyta. En L. Mora (Ed.), Catálogo ilustrado de las plantas de Cundinamarca. Bogotá: Volumen II, Instituto de Ciencias Naturales. Universidad Nacional de Colombia.

Murillo-P., M.T. (1968). Blechnum subgénero Blechnum en Suramérica con especial referencia a las especies de Colombia. Nova Hedwigia, 16, 329-366.

Murillo-P., M.T. (1983). Usos de los helechos en Sur América con especial referencia a Colombia. Bogotá: Instituto de Ciencias Naturales, Universidad Nacional de Colombia.

Murillo-P., M.T. (1986). Estudio preliminar del género Schizaea en Colombia. Caldasia, 15, 93-101.

Murillo-P., M.T. (1988a). Pteridophyta I. Bogotá: Flora de Colombia Volumen 2. Instituto de Ciencias Naturales. Universidad Nacional de Colombia. Imprenta Nacional.

Murillo-P., M.T. (1988b). Familia Hymenophyllaceae (Pteridófitos) del parque Nacional Isla de Gorgona. Revista de la Academia Colombiana de Ciencias Exactas, Físicas y Naturales, 16, 53-61.

Murillo-P., M.T. (1997). Los pteridófitos de la serranía de La Macarena. Caldasia, 19, 1-11.

Murillo-P., M.T. (2000). Helechos. En J. Rangel (Ed.), Colombia Diversidad Biótica III. La región de vida paramuna (pp. 528-562). Bogotá: Instituto de Ciencias Naturales, Universidad Nacional de Colombia.

Murillo-P., M.T. (2001). Acerca del "status" de Salpichlaena volubilis J. Smith in Hook. (Blechnaceae). Revista de la Academia Colombiana de Ciencias Exactas, Físicas y Naturales, 25, 229-236.

Murillo-P., M.T. \& Lozano, G. (1989). Hacia la realización de una flórula del Parque Nacional Natural Islas de Gorgona y Gorgonilla, Colombia. Revista de la Academia Colombiana de Ciencias Exactas, Físicas y Naturales, 17, 277-304.

Murillo-P., M.T. \& Harker, M. (1990). Helechos y plantas afines de Colombia. Bogotá: Colección Jorge Álvarez Lleras No. 2. Academia Colombiana de Ciencias Exactas, Físicas y Naturales.

Murillo-P., M.T. \& Murillo-A., J. (1999). Pteridófitos de Colombia I. Composición y distribución de las Lycopodiaceae. Revista de la Academia Colombiana de Ciencias Exactas, Físicas y Naturales, 23, 19-38.

Murillo-P., M.T. \& Murillo-A., J. (2001). Guía de pteridofitos (helechos y plantas afines) de Bogotá y sus alrededores. Bogotá: Impresol Ediciones Ltda.

Murillo-P., M.T. \& Murillo-A., J. (2004). Pteridofitos de Colombia V. El género Anemia (Schizaeaceae) en Colombia. Revista de la Academia Colombiana de Ciencias Exactas Físicas y Naturales, 28, 471-480.

Murillo-P., M.T. \& Smith, A.R. (2003). Luisma, a new genus of Grammitidaceae (Pteridophyta) from Colombia. Novon, 13, 313-317.

Murillo-P., M.T., Murillo-A., J. \& León, A. (2015).
Pteridófitos (Helechos y afines). En R. Bernal, S.R. Gradstein \& M. Celis (Eds.), Catálogo de plantas y líquenes de Colombia. Bogotá: Instituto de Ciencias Naturales, Universidad Nacional de Colombia, http:// catalogoplantasdecolombia.unal.edu.co.

Murillo-P., M.T., Murillo-A., J. \& León, A. (2016). Pteridófitos (Helechos y afines). En R. Bernal, S.R. Gradstein \& M. Celis (Eds.), Catálogo de plantas y líquenes de Colombia (pp. 446-578). Bogotá: Instituto de Ciencias Naturales, Universidad Nacional de Colombia.

Polanía, C., \& Murillo-A., J. (2004). Patrones de distribución espacial de Pteridófitos en la Amazonia colombiana. Acta Biológica Colombiana, 9, 127-128.

PPG I. (2016). A community-derived classification for extant lycophytes and ferns. Journal of Systematics and Evolution, 54, 563-603.

Ramírez, P. \& Macías, P. (2007). Catálogo de helechos y plantas afines del Departamento del Cauca. Popayán: Universidad del Cauca.

Ramírez, P. \& Mendoza, C. (2002). Monocotiledóneas y Pteridófitos de La Planada, Colombia. Biota Colombiana, 3, 285-295.

Rincón, A., Rangel-Ch, O. \& Aguirre, J. (2014). Musgos, líquenes, helechos y afines de la Orinoquia Colombiana. En: O. Rangel (Ed.), Colombia Diversidad Biótica XIV. La región de la Orinoquia de Colombia (pp. 419-446). Bogotá: Universidad Nacional de Colombia.

Rodríguez, W. (2002). Helechos, licopodios, selaginelas y equisetos del parque regional Arví. Medellín: Corporación Autónoma Regional de Antioquia. Editorial Multimpresos Ltda.

Rodríguez, W. (2011). Pteridófitas. En A. Idárraga-P., R.D.C. Ortiz, R. Callejas \& M. Merello (Eds.), Flora de Antioquia Catálogo de las plantas vasculares (pp. 134-204) Medellín: Volumen 2, Universidad de Antioquia.

Sanín, D., Mancera, J.C., Castaño, N., Alzate, N., González, G. \& Álvarez, L. (2006). Catálogo preliminar de las plantas vasculares de la reserva forestal protectora "Río Blanco" (Manizales, Caldas, Colombia). Boletín Científico-Centro de Museos Museo de Historia Natural, 10, 19-44.

Sanín, D., Álvarez, L. \& Castaño, N. (2008). Monilófitos y licófitos de la cuenca del río Chinchiná, Caldas, Colombia, Clave para géneros y catálogo de las especies. Revista Colombiana de Ciencias Exactas, Físicas y Naturales, 32, 331-352.

Schneider, H., Schuettpelz, E., Pryer, K., Cranfill, R., Magallón, S. \& Lupia, R. (2004). Ferns diversified in the shadow of angiosperms. Nature, 428, 553-557.

Toro, J.L. (2009). Estado del conocimiento de la flora silvestre en la jurisdicción de Corantioquia. Medellín: Corporación Autónoma Regional del Centro de Antioquia, Corantioquia.

Triana, L.A. \& Murillo-A., J. (2005). Helechos y plantas afines de Albán (Cundinamarca): El bosque subandino y su diversidad. Bogotá: Instituto de Ciencias Naturales, Universidad Nacional de Colombia Instituto Alexander Von Humboldt.

TRIANA, L.A. (2015a). Catálogo comentado de las 
especies de Pecluma (Polypodiaceae) de Colombia. Boletín Científico del Museo de Historia Natural de la Universidad de Caldas, 19, 17-59.

Triana, L.A. (2015b) Los helechos y licófitos de Caldas: Diagnóstico e identificación de prioridades de investigación. En J. Ramírez (Ed.), Libro de resumenes VIII Congreso Colombiano de Botánica ( $p$.
173). Manizales: Asociación Colombiana de Botánica. Tryon, R.M., \& Tryon, A. (1982). Ferns and allied plants. New York, NY: Springer-Verlag.

Zuloaga, F., Morrone, O. \& Belgrano, M. (Eds.) (2008). Catálogo de las plantas vasculares del Cono Sur: (Argentina, Sur de Brasil, Chile, Paraguay y Uruguay). Saint Louis: Missouri Botanical Garden Press. 